\title{
Relación entre insulinemia e hígado graso en pacientes con índice de masa corporal normal, sin antecedentes de consumo de alcohol crónico ni diabetes mellitus
}

\author{
Relationship between insulinemia and fatty liver \\ in patients with normal body mass index and \\ without a history of chronic alcohol consumption or \\ diabetes mellitus
}

\author{
William Sánchez, Quindo Santana, José Buelvas, Rafael Camargo • \\ BarRanquilla (Colombia)
}

\section{Resumen}

Existe una clara relación entre los pacientes con el hígado graso no alcohólico (HGNA) y la hiperinsulinemia, especialmente ante la presencia de factores de riesgo como la diabetes mellitus (DM), dislipidemia y obesidad; sin embargo, ésta puede presentarse incluso sin los factores de riesgo anteriormente mencionados.

Objetivo: determinar la relación existente entre HGNA, e hiperinsulinemia en pacientes con índice de masa corporal (IMC) normal y sin DM ni consumo crónico de alcohol.

Material y métodos: estudio descriptivo - transversal, tomando 59 pacientes adultos, 29 mujeres y 30 hombres en la Fundación Hospital Universitario Metropolitano (FHUM), sin antecedentes de consumo crónico de alcohol, con IMC normal y sin DM con las siguientes variables; la edad, sexo, perfil lipídico, ecografía hepática e insulinemia. Se utilizaron tablas de distribución de frecuencia, análisis multivariado con el riesgo relativo (RR) y Chi cuadrado $\left(\mathrm{X}^{2}\right)$, con un nivel de confianza del $95 \%$.

Resultados: en el análisis multivariado la correlación entre nivel de insulina mayor o igual de 8 UI, se encontró que la glucemia mayor de $90 \mathrm{mg} / \mathrm{L}$ fue de $53.8 \%$ (siete casos) e insulina menor de 8 con glucemia mayor de $90 \mathrm{mg} / \mathrm{L}$ fue el 43.5 (20 casos). Con un riesgo relativo 1.2246 (IC: 0.6460 - 2.3214); Chi cuadrado: $0.4315 \mathrm{p}=0.511$. Lo mismo con los niveles de insulina preestablecido con los siguientes resultados con un RR: 1.1047 (IC: 0.8655-1.4101) $\mathrm{X}^{2}: 1.0091 \mathrm{p}=0.3151$. Con una incidencia de $8.47 \%$ con hígado graso diagnosticado por ultrasonografía. En relación a la edad el promedio fue 24.5 años $( \pm 10.4)$.

Conclusiones: la relación de resistencia de la insulina y HGNA en este grupo de pacientes, muestran que la fuerza de la asociación RR se aleja de uno negativamente, lo que indica que no hay relación entre estas dos variables (Acta Med Colomb 2011; 36: 73-77).

Palabras clave: hígado graso no alcohólico, hiperinsulinemia, diabetes mellitus dislipidemia, obesidad.

\footnotetext{
Abstract

There is a clear relationship between patients with nonalcoholic fatty liver disease (NAFLD) and hyperinsulinemia, especially in the presence of risk factors like diabetes mellitus (DM), dyslipidemia, and obesity. However, this can occur even without the risk factors mentioned above.

Objective: to determine the relationship between NAFLD, and hyperinsulinemia in patients with normal body mass index (BMI) and without DM or chronic alcohol consumption.

Materials and methods: cross, taking 59 adult patients, 29 women and 30 men in the Metropolitan University Hospital Foundation (FHUM) with no history of chronic alcohol consumption, with normal
}

Dr. William Sánchez Rincones: Coordinador de Posgrado de Medicina Interna, Universidad Metropolitana; Dr. Quindo Santana Fontalvo: Residente II año de Medicina Interna, Universidad Metropolitana; Dr. José Buelvas Díaz:Residente I año de Medicina Interna, Universidad Metropolitana; Rafael Camargo de Mier: M.Ed. Asesor Metodológico. Universidad Metropolitana. Barranquilla (Colombia) Correspondencia. Dr. William Sánchez E-mail: wsr7@hotmail.com Recibido: 20/XII/2010 Aceptado: 04/V/2011 
BMI and without DM with the following variables: age , sex, lipid profile, liver ultrasound and insulin. We used frequency distribution tables, multivariate analysis the relative risk (RR) and Chi square test with a confidence level of $95 \%$.

Results: in multivariate analysis the correlation between insulin level greater or equal to 8 IU, blood glucose was found that greater than $90 \mathrm{mg} / \mathrm{L}$ was $53.8 \%$ ( 7 cases) and insulin with glucose levels less than 8 greater than $90 \mathrm{mg}$ / L was 43.5 (20 cases). 1.2246 with a relative risk (CI: 0.6460 to 2.3214), Chi square: $\mathrm{p}=0.5110 .4315$. Same with preset insulin levels with the following results with a relative risk: 1.1047 (CI 0.8655 to 1.4101 ) Chi square: $\mathrm{p}=0.31511 .0091$. With an incidence of $8.47 \%$ with fatty liver diagnosed by ultrasonography. Regarding the average age was 24.5 years $( \pm 10.4)$.

Conclusions: the relationship of insulin resistance and NAFLD in this group of patients showed that the strength of the association RR away from a negative, which indicate no relationship between these two variables (Acta Med Colomb 2011; 36: 73-77).

Keywords: nonalcoholic fatty liver, hyperinsulinemia, dyslipidemia, diabetes mellitus, obesity.

\section{Introducción}

El reto para los profesionales de la salud es grande, no sólo en la prevención de las enfermedades crónicas relacionadas con lo alimentario y nutricional, como el hígado graso no alcohólico (HGNA) y sus patologías asociadas, sino con el mantenimiento de la salud desde las etapas tempranas de la vida. La enfermedad HGNA es una entidad clínicopatológica reconocida en las últimas décadas. Se define como la presencia de esteatosis hepática en más de $5 \%$ de los hepatocitos, con o sin la presencia de inflamación y fibrosis, en un paciente con una ingesta alcohólica menor de $40 \mathrm{~g}$ a la semana e idealmente, sin otras causas de daño hepático crónico.

El HGNA caracterizado por la presencia de varios de los problemas relacionados con la resistencia a la insulina (IR), demanda de un tratamiento ajustado a las necesidades de cada paciente, en el que interactúan dieta, actividad física y de ser necesario tratamiento temprano o preventivo. No hay una dieta única para el tratamiento del HGNA, es relevante identificar cada alteración metabólica y hacer recomendaciones dietarías específicas y contextualizadas a la situación del paciente.

La IR se encontraría presente aun en los pacientes no obesos y el riesgo de esteatosis aumenta cuando se asocia a un mayor número de componentes del síndrome metabólico $(1,2)$. Algunos estudios han demostrado una alta prevalencia de intolerancia a glucosa y diabetes en pacientes con HGNA $(3,4)$, y su presencia aumentaría el riesgo de progresión a fibrosis (5).

La enfermedad por hígado graso no alcohólico (EHGNA), se define como la presencia de esteatosis hepática en más de 5\% de los hepatocitos, con o sin la presencia de inflamación y fibrosis, en un paciente con una ingesta alcohólica menor de $40 \mathrm{~g}$ a la semana e idealmente, sin otras causas de daño hepático crónico. La prevalencia, según algunas series extranjeras, es de alrededor de $20 \%$ en la población general $(6,7)$.

Clásicamente descrito en grupos de riesgo diabéticos, hipertrigliceridémicos u obesos, hoy se reconoce que el HGNA puede darse en personas sin factores de riesgo aparentes (8).
La esteatohepatitis no alcohólica (EHNA) debe considerarse integrada en una entidad de espectro más amplio, el hígado graso no alcohólico (HGNA), que abarca desde la esteatosis hepática como forma inicial, pasando por la EHNA propiamente dicha, que puede evolucionar a una cirrosis hepática, a menudo filiada como criptogenética por haber perdido sus características específicas, que se sitúa en el otro extremo del espectro. La EHNA es una enfermedad crónica muy frecuentemente en pacientes con alteración de la función hepática. La mayoría son pacientes obesos, mujeres y con diabetes mellitus del tipo 2 y/o hiperlipidemia y resistencia a la insulina. Sin embargo, también se presenta en varones y mujeres delgados sin patologías asociadas y en relación con una miscelánea de situaciones como cirugía derivativa, toma de fármacos, etc. Powell y cols. (9) definen la EHNA como la presencia de hallazgos histológicos característicos, elevación de transaminasas, sin antecedentes de ingesta significativa de alcohol $(<30 \mathrm{~g}$ a la semana confirmado por la familia) y ausencia de otro tipo de hepatopatías.

La esteatohepatitis no alcohólica (EHNA) es un estadio intermedio en el espectro de la enfermedad hepática grasa no alcohólica (EHGNA), una enfermedad hepática crónica que histológicamente recuerda a la hepatitis alcohólica, pero que ocurre en individuos que no son bebedores. Es la causa más frecuente de elevación inespecífica de transaminasas en la práctica diaria, y la evidencia que la señala como etiología de la cirrosis "criptogénica" es cada vez mayor. Como en la esteatosis alcohólica, la EHGNA varía desde la esteatosis en el extremo más benigno del espectro hasta la cirrosis en el extremo opuesto, donde la mayoría de morbilidad y mortalidad asociada a enfermedad hepática ocurre (10).

Los últimos estudios relacionados con hígado graso asociados a esteatosis hepática no alcohólica, vinculan a la hiperinsulinemia como factor hormonal preponderante en el compromiso fisiopatológico del mismo, y elemento desencadenante de una serie de alteraciones que conllevan a mayor deterioro orgánico y empobrecimiento del pronóstico.

Por otro lado, la hiperinsulinemia en asociación a resistencia periférica a la insulina e hiperglucemia, se encuentra 
relacionada con riesgo cardiovascular y metabólico, como lo confirman los últimos estudios sobre obesidad, diabetes mellitus, síndrome metabólico, determinando un valor estadístico significativo sobre la morbimortalidad de estos síndromes y enfermedades en cuya virtud se establecen parámetros y aproximaciones pronósticas, basadas en múltiples factores de riesgo, todos ellos en asociación como lo hacen los puntajes establecidos como riesgo pronóstico de mortalidad por evento cardiocerebrovascular a 10 años, para hombres y mujeres (Framingham).

Siendo evidente la relación entre insulina con hígado graso por esteatosis hepática no alcohólica, así como con múltiples alteraciones fisiopatológicas relacionadas con riesgo cardiovascular, como obesidad abdominal, hiperglucemia, hipertensión arterial, resistencia periférica a la insulina e hiperlipidemia, entre otras, consideramos de vital importancia establecer en aquellos pacientes diagnosticados con hígado graso aislado, en los cuales no se encuentran antecedentes de diabetes mellitus, alcoholismo ni obesidad, la presencia de hiperinsulinemia como factor de riesgo básico para desarrollar de forma potencial, todos aquellos elementos inherentes al síndrome metabólico, permitiendo de esta manera iniciar en forma precoz cambios en el estilo de vida y medidas de prevención a pesar de encontrarnos frente a un paciente sin aparentes factores de riesgo cardiovasculares.

Motivados en determinar qué tanta relación se puede encontrar entre el hígado graso no alcohólico y la insulina como hormona vinculada al mismo, y ante la poca información bibliográfica sobre este tópico, planteamos el siguiente interrogante:

¿Existe relación entre hígado graso y el nivel de insulinemia, en pacientes con índice de masa corporal normal, sin antecedentes de diabetes mellitus ni alcoholismo?

Por tales razones, el objetivo del presente trabajo fue determinar la relación existente entre hígado graso, e hiperinsulinemia en pacientes no alcohólicos, con índice de masa corporal (IMC) normal y sin antecedentes de diabetes mellitus ni alcoholismo.

\section{Tipo de estudio}

\section{Material y métodos}

Descriptivo transversal del comportamiento de la insulinemia e hígado graso en pacientes con índice de masa corporal normal, sin antecedentes de alcoholismo ni diabetes mellitus.

\section{Población y muestra}

Conformada por pacientes adultos, con índice de masa corporal normal, sin antecedentes de alcoholismo ni diabetes mellitus.

\section{Variables}

Fueron primordiales para el estudio la edad, sexo, perfil lipídico, ecografía hepática e insulinemia.

\section{Fuente de la información}

Formato propuesto por el proyecto, se tomó como fuentes secundarias, el registro y las bases de datos del área ecografía y laboratorio.

\section{Plan de recolección y análisis de la información}

Para la recolección de información se empleó un formato que garantizó el orden del mismo a fin de facilitar su clasificación, digitación y tabulación.

La información se tabuló por medios computarizados, utilizando la base de datos del programa Epi Info 5.3.1 y el programa Excel 2000, los cuales se emplearon para la realización de las tablas con sus estadísticas y los gráficos respectivamente.

Se tomaron todos adultos con índice de masa corporal normal, sin antecedentes de alcoholismo ni diabetes mellitus. El fin de establecer la prevalencia de insulinemia e hígado graso, su relación con los determinantes como estado de la glucemia y el perfil lipídico.

Se realizaron tablas de distribución de frecuencia, con su análisis de frecuencia, porcentaje, tasa, y medidas de tendencia central y dispersión, de acuerdo con la naturaleza de las variables para el análisis univariado y para el análisis multivariado el riesgo relativo (RR) y $\mathrm{X}^{2}$.

\section{Resultados}

Se evaluaron 59 pacientes, $50.8 \%$ de sexo femenino (29) y $49.9 \%$ de sexo masculino (30) con una incidencia de $8.47 \%$ con hígado graso diagnosticado por ultrasonografía. En relación con la edad el promedio fue de 24.5 años $( \pm 10.4)$, con un mínimo de 18 y un máximo de 44 . El promedio para la glucemia fue de $91.7( \pm 23.2)$, moda de 84 y un mínimo de 69 y máximo de 119 (Figura 1).

El grupo etáreo más estudiado fue el de 21-30 años con un 79.7\%, siguiéndole el de 18-20 con un 15.3\% (Tabla 1).

La distribución porcentual de los niveles de colesterol del grupo mayor de $200 \mathrm{mg} / \mathrm{dL}$ fue de $28.8 \%$ (17 casos), con un intervalo de confianza al $95 \%$ de $17.8 \%$ a $42,1 \%$. El valor promedio para colesterol fue de $186.7( \pm 84.5)$ y con mínimo de $120 \mathrm{mg} / \mathrm{dL}$ y un máximo de $307 \mathrm{mg} / \mathrm{dL}$ (Tabla 2).

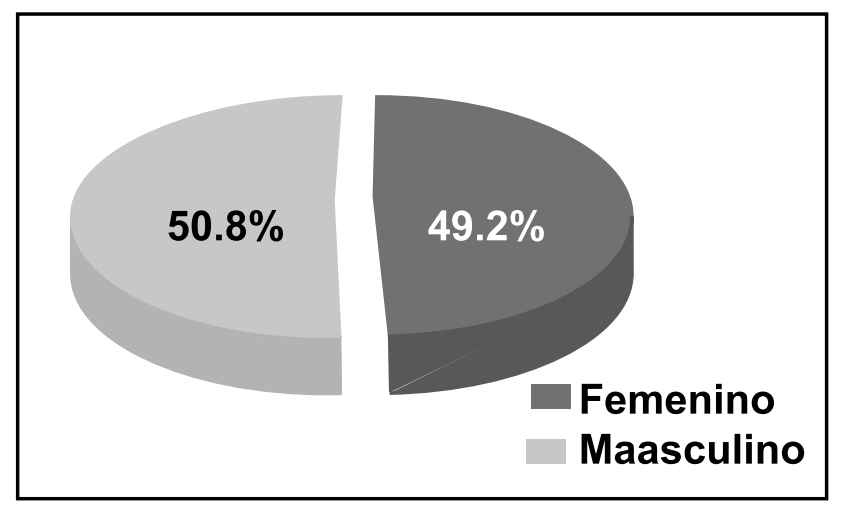

Figura 1. Distribución de pacientes según sexo. Fuente: Reportes de datos tomados por el investigador, Barranquilla, 2008. 
Tabla 1. Distribución de pacientes según grupo de edad.

\begin{tabular}{|l|c|c|}
\hline Edad & Frecuencia & Porcentaje (\%) \\
\hline $18-20$ & 9 & 15.30 \\
$21-30$ & 47 & 79.70 \\
$31-40$ & 1 & 1.70 \\
$41-44$ & 2 & 3.40 \\
\hline Total & $\mathbf{5 9}$ & $\mathbf{1 0 0 . 0 0}$ \\
\hline Fuente: Reportes de datos tomados por el investigador, Barranquilla, 2008. \\
\hline
\end{tabular}

Tabla 2. Distribución porcentual de niveles de colesterol.

\begin{tabular}{|l|c|c|}
\hline $\begin{array}{l}\text { Nivel de } \\
\text { colesterol }\end{array}$ & Frecuencia & $\begin{array}{c}\text { Intervalo de } \\
\text { confianza al 95\% }\end{array}$ \\
\hline$\leq 200 \mathrm{mg} / \mathrm{dL}$ & $42(71.2 \%)$ & $57.9 \%-82.2$ \\
$>200 \mathrm{mg} / \mathrm{dL}$ & $17(28.8 \%)$ & $17.8 \%-42.1$ \\
\hline Total & $\mathbf{5 9 ( 1 0 0 . 0 \% )}$ & \\
\hline Fuente: Reportes de datos tomados por el investigador, Barranquilla, 2008. \\
\hline
\end{tabular}

Tabla 3. Distribución porcentual de niveles de triglicéridos.

\begin{tabular}{|l|c|c|}
\hline $\begin{array}{l}\text { Nivel de } \\
\text { triglicérido }\end{array}$ & Frecuencia & $\begin{array}{c}\text { Intervalo de } \\
\text { confianza al 95\% }\end{array}$ \\
\hline$\leq 150 \mathrm{mg} / \mathrm{dL}$ & $34(57.6 \%)$ & $44.1 \%-70.4$ \\
$>150 \mathrm{mg} / \mathrm{dL}$ & $25(42.4 \%)$ & $29.6 \%-55.9$ \\
\hline Total & $\mathbf{5 9 ( 1 0 0 . 0 \% )}$ & \\
\hline \multicolumn{2}{|c|}{ Fuente: Reportes de datos tomados por el investigador, Barranquilla, 2008. } \\
\hline
\end{tabular}

Tabla 4. Distribución porcentual de pacientes con hipercolesterinemia según con y sin hígado graso.

\begin{tabular}{|l|c|c|c|}
\hline \multirow{2}{*}{$\begin{array}{l}\text { Nivel de } \\
\text { colesterol }\end{array}$} & \multicolumn{2}{|c|}{ Ecografía } & Total \\
\cline { 2 - 4 }$\leq 200 \mathrm{mg} / \mathrm{dL}$ & $39(92.9 \%)$ & $3(7.1 \%)$ & $42(71.2 \%)$ \\
$>200 \mathrm{mg} / \mathrm{dL}$ & $15(88.2 \%)$ & $2(11.8 \%)$ & $17(28.8 \%)$ \\
\hline Total & $\mathbf{5 4}(\mathbf{9 1 . 5 \% )}$ & $\mathbf{5 ( 8 . 5 \% )}$ & $\mathbf{5 9}(\mathbf{1 0 0 \% )})$ \\
\hline Riesgo relativo: 1.0524 (IC: $0.8679-1.2761)$. \\
$\mathrm{X}^{2}: 0.3276 \mathrm{P}=0.5670$.
\end{tabular}

Tabla 5. Distribución porcentual de pacientes con hipertrigliceridemia y según con y sin hígado graso.

\begin{tabular}{|c|c|c|c|}
\hline \multirow{2}{*}{$\begin{array}{l}\text { Nivel de } \\
\text { triglicéridos }\end{array}$} & \multicolumn{2}{|c|}{ Ecografía } & \multirow[t]{2}{*}{ Total } \\
\hline & Normal & H.G.N.A. & \\
\hline$\leq 150 \mathrm{mg} / \mathrm{dL}$ & $30(88.2)$ & $4(11.8 \%)$ & $34(57.6 \%)$ \\
\hline$>150 \mathrm{mg} / \mathrm{dL}$ & $24(96.0 \%)$ & $1(4.0 \%)$ & $25(42.4 \%)$ \\
\hline Total & $54(91.5 \%)$ & $5(8.5 \%)$ & $59(100 \%)$ \\
\hline \multicolumn{4}{|c|}{$\begin{array}{l}\text { Riesgo relativo: } 0.919 \text {. } \\
\text { (IC: } 07939-1.0642 \text { ). } \\
\mathrm{X}^{2}: 1.1009 \mathrm{p}=0.2940\end{array}$} \\
\hline
\end{tabular}

El nivel de triglicéridos mayor de $150 \mathrm{mg} / \mathrm{dL}$ fue de $42.4 \%$ ( 25 casos) con un intervalo de confianza al $95 \%$ de $29.6 \%$ a $55.9 \%$ con una valor promedio $144.3( \pm 135.4)$, un mínimo de 45 y un máximo de 338 (Tabla 3 ).

En el análisis multivariado la correlación nivel de insulina mayor o igual 8 UI se encontró que la glucemia mayor de $90 \mathrm{mg} / \mathrm{dL}$ fue de $53.8 \%$ (siete casos) e insulina menor de ocho con glucemia mayor de $90 \mathrm{mg} / \mathrm{dL}$ fue el 43,5 (20 casos). Con un riesgo relativo 1.2246 (IC: 0.6460-2.3214); Chi cuadrado: $0.4315 \mathrm{p}=0.5112$; lo mismo con los niveles de insulina preestablecido con HGNA e hígado normal con los siguientes resultados con un riesgo relativo: 1,1047 (IC: 0.8655-1.4101) Chi cuadrado: $1.0091 \mathrm{p}=0.31513$ (Tablas 4, 5).

\section{Discusión}

La relación de resistencia de la insulina y HGNA en este grupo de pacientes, arrojó que en este trabajo la fuerza de la asociación RR se aleja de uno negativamente, lo que indica que no hay relación entre estas dos variables.

El resultado de pruebas bioquímicas hipercolesterinemia 17 (29.3\%), hipertrigliceridemia 17 (29.3\%) como factor de riesgo en el HGNA difiere de la fisiopatología descrita manifestando que ésta se asocia a alteración en el estrés oxidativo, en la actividad de la citocromo $\mathrm{P} 450$ y en algunos casos liberación de mediadores inflamatorios dentro del hepatocito que induce a una alteración en el metabolismo de los lípidos y carbohidratos, lo cual no es coherente con los resultados encontrados. Así mismo en el cruce de colesterolemia y HGNA el intervalo de confianza que pasó por uno y la $\mathrm{P}=0,86$ niega la significancia estadística de esta asociación e igual para los demás cruces trigliceridemia, insulinemia y glucemia.

En el análisis multivariado de la interrelación o asociación de las variables insulina y glucemia, insulina e hígado graso no alcohólico, dislipidemia e hígado graso no alcohólico el riesgo relativo calculado, los intervalos de confianza y los valores de (p) no se halló asociación ni significancias estadística (Tablas 4, 5).

Por último, de los cinco pacientes sólo dos presentaron alteración del perfil lipídico y de los que presentaron alteración, 15 no tenían hígado graso, de lo que podemos afirmar debe haber otra condición más que altere la respuesta del hígado a la lipidemia, una HGNA que no puede ser secundaria a hepatitis, abuso de alcohol, exposición a tóxicos, enfermedad autoinmune, hepatopatía congénita, obstrucción vascular o enfermedad del tracto biliar.

\section{Conclusiones}

Con base en el sexo, se encontró que 50.8\% corresponde al femenino (29) y $49.9 \%$ al masculino (30).

La incidencia de hígado graso diagnosticado por ultrasonografía fue $8.47 \%$.

La edad promedio fue 24.5 años, con un mínimo de 18 y un máximo de 44. 
El grupo etario más estudiado fue el de 21-30 años con $79.7 \%$, seguido por el de $18-20$ con $15.3 \%$.

El promedio de glucemia fue $91.7 \mathrm{mg} / \mathrm{dL}$, la moda de 84 con un mínimo de 69 y un máximo de 119.

La distribución porcentual de los niveles de colesterol mayor de $200 \mathrm{mg} / \mathrm{dL}$ fue $29.3 \%$ (17 casos).

El nivel de triglicéridos mayor de $150 \mathrm{mg} / \mathrm{dL}$ fue $40 \%$ (22 casos).

Ninguno presentó insulina ni glucemia por encima de los estándares establecido; no hubo relación HGNA y resistencia a la insulina.

\section{Referencias}

1. Marceau P, Biron S, Hould FS, Marceau S, Simard S, Thung SN, et al. Liver pathology and the metabolic syndrome $\mathrm{X}$ in severe obesity. J Clin Endocrinol Metab 1999; 84: 1513-7.

2. Marchesini G, Brizi M, Bianchi G, Tomassetti S, Bugianesi E, Lenzi M, et al.
Nonalcoholic fatty liver disease. A feature of the metabolic syndrome. Diabetes 2001; 50: 1844-50.

3. Willner IR, Waters B, Patil SR, Reuben A, Morelli J, Riely CA. Ninety patients with nonalcoholic steatohepatitis: insulin resistance, familial tendency, and severity of disease. Am J Gastroenterol 2001; 96: 2957-61.

4. Gerber T, Schomerus L. Hepatic encephalopathy in liver cirrhosis. Drugs 2000; 60: $1252-1370$

5. Uribe M, Guevara L. Encefalopatia Hepatica.En : Berenguer J,editor. Gastroenterologia y Hepatologia.3ra.edicion Barcelona :Editorial Doyma; 2002: 789-803.

6. Bellentani S, Tiribelli C, Saccoccio G, Sodde M, Fratti N, De Martin C, et al. Prevalence of chronic liver disease in the general population of Northern Italy: the Dionysis study. Hepatology 1994; 20: 1442-9.

7. Wanless IR, Lentz JS. Fatty liver hepatitis (steatohepatitis) and obesity: Autopsy study with analysis of risk factors. Hepatology 1990; 12: 1106-10.

8. Bacon BR, Farahvash MJ, Janney CG, Neuschwander-Tetri BA. Nonalcoholic steatohepatitis: An expanded clinical entity. Gastroenterology 1994; 107:1103-9.

9. Powell EE, Cooksley WGE, Hanson R, Searle J, Halluday JW, Powell LW. The natural history of nonalcoholic steatohepatitis: a follow-up study of forty-two patients for up to 21 years. Hepatology 1990; 11: 74-80.

10. Brunt EM. Non alcoholic Steatohepatitis. Semin Liv Dis 2004; 24: 3-20. 